\title{
A controlled, randomized nonblinded clinical trial to assess the efficacy of amphotericin B deoxycholate as compared to pentamidine for the treatment of antimony unresponsive visceral leishmaniasis cases in Bihar, India
}

\author{
Vidya Nand Rabi Das' \\ Niyamat Ali Siddiqui ${ }^{2}$ \\ Krishna Pandey ${ }^{3}$ \\ Vijay Pratap Singh ${ }^{2}$ \\ Roshan K Topno ${ }^{4}$ \\ Dharmendra Singh ${ }^{5}$ \\ Rakesh Bihari Verma ${ }^{2}$ \\ Alok Ranjan ${ }^{2}$ \\ Prabhat Kumar Sinha' \\ Pradeep Das ${ }^{6}$ \\ 'Department of Clinical Medicine, \\ Rajendra Memorial Research Institute \\ of Medical Sciences, Indian Council \\ of Medical Research, Patna, Bihar, \\ India; ' ${ }^{2}$ Department of Biostatistics; \\ ${ }^{3}$ Department of Clinical Medicine; \\ ${ }^{4}$ Department of Epidemiology; \\ ${ }^{5}$ Department of Molecular Biology; \\ ${ }^{6}$ Director and Institutional Head, \\ Rajendra Memorial Research Institute \\ of Medical Sciences, Indian Council of \\ Medical Research, Patna, Bihar, India
}

Correspondence: Vidya N R Das Rajendra Memorial Research, Institute of Medical Sciences (I.C.M.R.), Govt of India, Ministry of Health and F.W., Agam-Kuan, Patna-800007 (Bihar), India $\mathrm{Tel}+9|6| 2263|56|$

$\mathrm{Fax}+916122634379$

Email dirrmris@sancharnet.in

\begin{abstract}
Background: There is significant variation in Amphotericin B (AMB) efficacy and relapses in antimony unresponsive visceral leishmaniasis (VL) cases over a period of time (10-15 years). Keeping in mind the above mentioned view this study was undertaken with an objective to assess the magnitude of cure and relapse rates of AMB in the treatment of antimony unresponsive VL cases.
\end{abstract}

Methods: In a controlled, randomized nonblinded clinical trial, we evaluated the cure and relapse rate of Amphotericin B deoxycholate as compared to pentamidine. A total of 82 sodium stibogluconate (SSG) unresponsive and parasitologically confirmed VL cases were included in this study and randomized into two groups, test (Amphotericin B) and control (Pentamidine). Both the groups were treated with recommended dosages (as per World Health Organization guidelines) of respective medicines. All the patients were followed up on 1st, 2nd, and 6th month after end of treatment.

Results: Apparent cure rate in the Amphotericin B group was found to be 95\% (39/41) compared with $83 \%$ (34/41) in the Pentamidine group, which shows significant statistical difference ( $p=0.05$ ). The ultimate cure rate was found $92 \%$ (38/41) in the Amphotericin B group compared to $73 \%$ (30/41) in the Pentamidine group, which shows a significant statistical difference (Yates corrected chi-square $=4.42, p=0.04$ ). Similarly, significant statistical difference was observed in the relapse rate of the Amphotericin group compared to the Pentamidine group ( $p=0.03)$.

Conclusions: AMB may still be the drug of choice in the management of resistant VL cases in Bihar, India. This is due to its consistent apparent cure rate (95\%), low relapse rate (2.5\%), and cost effectiveness compared with other available antileishmanial drugs. It is a safe drug even in case of pregnancy. Efforts should be taken to form a future strategy so that this drug and coming newer drugs do not meet a similar fate as has happened to SSG and pentamidine over a span of $10-15$ years.

Keywords: sodium stibogluconate, apparent cure rate, ultimate cure rate, relapse rate, Amphotericin B deoxycholate

\section{Introduction}

Visceral leishmaniasis (VL) (Kala-azar) is a disease caused by a protozoan parasite, Leishmania donovani and transmitted by the bite of sandfly vector Phlebotomus argentipes. It occurs in more than 80 countries in Asia, Africa, Southern Europe, and South America, with a total of 200 million people at risk. ${ }^{1,2}$ However $90 \%$ of the estimated 500,000 new symptomatic cases per year occur in just five countries: India, Sudan, Bangladesh, Nepal, and Brazil. ${ }^{3}$ India contributes to about $40 \%-50 \%$ of the 
world's total cases, from which $90 \%$ are from Bihar State alone. Kala-azar remains a serious public health problem in Bihar. ${ }^{4}$ The present treatment scenario of Kala-azar in Bihar is extremely unsatisfactory. The rising trend of unresponsiveness to sodium stibogluconate (SSG) in Bihar has been reported as $35 \%-45 \%$. This has resulted in loss of affordable drugs to the region and forced clinicians to use alternative antileishmanial drugs. ${ }^{5-7}$ Pentamidine isethionate was the first drug tried as second-line treatment. This was widely used at district hospitals in endemic areas of Kala-azar in Bihar till three to four years ago. But due to diminishing efficacy ( $80 \%$ ) over a period of time, serious toxicity such as insulindependent diabetes, and cardiac toxicity in addition to its cost limited its use. ${ }^{8-10}$ Further reviews reveal the quick decline of response rate of pentamidine from $>95 \%$ in the early $1980 \mathrm{~s}$ to $<70 \%$ a decade later. ${ }^{11,12}$ Numerous oral agents have been tested and discarded. ${ }^{13-15}$ Several combination therapies have also been tried. The result of therapeutic advances is miltefosine, an oral drug which seems to be a step in the right direction as shown by multicenter clinical trials in India. ${ }^{16}$ Many other injectable/oral drugs are in trial stages. Presently Amphotericin B (AMB) is the only available alternative treatment of choice in the Indian Government set-up in Bihar. But unfortunately significant variation in AMB efficacy was reported in antimony unresponsive cases over a period of time. Prior conducted studies have reported efficacy in the range of $98 \%-100 \% .{ }^{17-19}$ Further review of literature reveals that the AMB efficacy has fallen to $90 \%-93 \%$ in India as well as in other parts of the world. ${ }^{20,21}$ Recent reviews reveal a cure rate of about $96 \%-97 \%$, at the sixth month in either daily or alternate day infusions. ${ }^{22,23}$ Earlier reviews advocated AMB as a first-line drug in the treatment of both unresponsive and fresh cases of Kala-azar. ${ }^{23}$ Keeping in view the burden of the disease in poorer sections of India, widespread emergence of drug resistance, recent availability of an oral drug (miltefosine) and the Indian Government commitment to eliminate Kala-azar, the present study was undertaken to assess the magnitude of cure rate and relapse rate of AMB in the treatment of antimony unresponsive Kala-azar cases in Bihar over a period of the last 15 years.

\section{Materials and methods}

\section{Study sites}

Rajendra Memorial Research Institute of Medical Sciences (RMRIMS) is situated at Patna, the capital of Bihar state of India on the banks of the holy river, Ganges. RMRIMS is a research institute that focuses on Kala-azar research and treatment. The Institute has 50-bed indoor and outdoor facilities and is a recognized specialized treatment centre for Kala-azar. Generally, patients are referred from various primary health centers and district hospitals with their earlier treatment records to RMRIMS in cases of failure to respond to the first-line drug SSG at the periphery level. They were screened for inclusion and exclusion criteria of the trial at the time of attending the outpatient department (OPD). They were included in the study after fulfilling the inclusion and exclusion criteria. The present study was conducted in the year 2002 and follow-ups were completed in the same year.

\section{Study patients}

Patients unresponsive to SSG after full course of treatment and having complaints of continued fever with rigor, hepato-splenomegaly, anorexia, anemia, loss of weight and attending RMRIMS OPD were the study patients. Their treatment records were verified at the time of inclusion in the study group.

\section{Treatment allocation}

Treatment allocation was done by the biostatistician of the institute, who performed the allocation sequence using random number tables and accordingly assigned the test and control group. The patients were sent to the indoor ward for further treatment with their allotted drug. Both groups of patients were treated after the hospitalization in RMRIMS indoor ward. Every patient completed the full course of assigned treatment.

\section{Inclusion criteria}

Patients of either sex aged between six to 60 years unresponsive to SSG after a full course of treatment and having complaints of continued fever with rigor, hepato-splenomegaly, anorexia, anemia, and loss of weight, sputum for acid-fast bacilli (AFB) and chest x-ray (PA-view) negative for tuberculosis or pneumonia, and demonstration of parasite in bone marrow (BM)/splenic(SPL) aspiration were the inclusion criteria.

\section{Exclusion criteria}

The confirmed VL cases with partial dosages of SSG and with associated diseases like tuberculosis (TB), HIV/AIDS, and malaria, pregnant women, lactating mother, and diabetics were not included in the study. The individuals who had past history of Kala-azar in last five years and having $\mathrm{Hb}<6 \mathrm{~g} / \mathrm{dl}$, platelet count $<50,000 / \mathrm{mm}^{3}$ with significant abnormalities in liver and renal function tests were the exclusion criteria. 
It is documented that if $\mathrm{Hb}<6 \mathrm{mg} / \mathrm{dl}$ and platelet count $<50,000 / \mathrm{mm}^{3}$ there is a possibility of reduced efficacy of the drug as well as its increased toxicity. ${ }^{24}$

\section{Informed consent}

Written informed consents for treatment and follow-up examinations were obtained from adult subjects and in case of minors, assent form was obtained from the legal representative of the subject prior to enrollment in the study.

\section{Sample size}

The hypothesis for the current study was to assess whether the cure and relapse rate of $\mathrm{AMB}$ was consistent as compared to pentamidine. We believed that the reported cure rate of AMB (based on the literature) would be $95 \%$ in the Amphotericin B group, while it would be about $75 \%$ in the Pentamidine group. Based on $90 \%$ power to detect a significant difference $(\mathrm{P}=0.05$, two-sided 100 patients were required for the study. To compensate for a $10 \%$ drop out, an optimum sample size for this clinical trial was 110 patients, which came to 55 patients in each group. A total of only 82 (41 to each group) SSG-unresponsive and parasitologically confirmed Kala-azar cases satisfied inclusion and exclusion criteria and were included in the present study. The required sample size of 55 could not be achieved under the study because the pentamidine supply was stopped by the Government of India in the mean time.

\section{The study intervention}

The test group was treated with AMB ( $1 \mathrm{mg} / \mathrm{kg}$ body weight on alternate days in 5\% dextrose over a period of 3-4 hours, intravenously for 15 days, starting with test dose) whereas the control group was treated with pentamidine $(4 \mathrm{mg} / \mathrm{kg}$ body weight on alternate days with deep intramuscular injections for 15 days).

\section{Investigations}

Complete physical examination and clinical findings were recorded in a pre-designed proforma. Laboratory investigations included a complete hemogram, blood biochemistry including electrolyte estimation and liver and kidney function tests. A chest radiogram and electrocardiogram were done. Splenic/BM aspiration was done when PT (prothrombin time) value was within normal limit and examined under microscope for Leishman-Donovan bodies. In cases of VL, who had severe anemia $(\mathrm{Hb}<6 \mathrm{gm} / \mathrm{dl})$, before excluding such patient from the study an effort was undertaken to increase their hemoglobin level by giving fresh blood transfusion as required. All laboratory investigations except blood sugar mentioned above were repeated on 14 th day after the initiation of treatment and at the end of treatment, while blood glucose was monitored on 0, 7, 14 days and at the end of treatment and during follow-up to observe the main complication of pentamidine, ie, diabetes mellitus. All vital signs (such as blood pressure, pulse, respiration rate, body temperature, etc.) were monitored daily. Specific clinical examinations (like measurement of spleen size and liver size) were done on every 7 th day till the end of treatment. Parasitological examination was done before and after completion of therapy as well as at the end of six months follow-up in relapse cases. All the patients completed the full course of their assigned treatment and none were shifted to any other drug, even after minor toxicity which was managed accordingly. As soon as their blood investigation came to normal, treatment was restarted to complete the course. Those patients who were excluded from the study were referred to the Medical College for evaluation and further treatment. Rescue treatment was given in case of drug-induced toxicity and follow-up was carried out for six months after end of treatment.

\section{Adverse reaction}

Anaphylactic reactions ie, sweating, breathlessness, low pulse rate etc. were closely monitored clinically by the treating physician and staff nurses; recorded in the patient record sheet and such events were corrected simultaneously with $25 \%$ dextrose infusion and with antiallergic drugs in the Pentamidine group. The occurrence of adverse reactions was monitored and characterized for each of the regimens. Minor adverse reactions of both the groups were managed as per the standard normal procedures.

\section{Follow-up}

All the patients were followed up on the 1st, 2nd, and 6th month after end of treatment. Patients were instructed to report immediately in case of fever or other complications. The patients were called for follow up on due date and if they failed a postcard was sent on their home addresses. If they did not turn up, individual home visits were undertaken. Clinical examinations and routine laboratory investigations were done at each follow up. The BM aspiration was done for parasites at final follow up in cases of relapse. All the patients completed six months follow up.

\section{Apparent cure}

Apparent cure was defined as absence of clinical signs and symptoms for Kala-azar as well as parasitologically negative 
for Leishmania donovani parasite in their SPL/BM aspiration examination at the end of treatment.

\section{Ultimate cure}

Ultimate cure was defined as absence of clinical signs and symptoms for VL at the end of six months follow-up after end of treatment.

\section{Relapse}

Relapse was defined as reappearance of clinical signs, symptoms such as intermittent fever, enlargement of liver, enlargement of spleen and Leishmania donovani parasite in SPL/BM aspiration within a period of six months during follow-up.

\section{Data analysis}

Data were coded as numerical variables, entered into, and analyzed using the Epi-Info 6.0 database (Centre for Disease Control and Prevention, Atlanta, GA). Comparison of the two treatment groups and their baseline characteristics were done, using large sample test based on Gaussian distribution. Yates's corrected Chi-square test was used for comparing the efficacy and relapse rate of the two regimens.

\section{Ethical considerations and consent}

The study was approved by the scientific advisory committee and by the ethical committee of the Institute. Informed consent written in Hindi was obtained from each patient or guardian. If necessary, consent was verbally translated into the local language. Decision regarding management of patients were based on their clinical condition and were independent of the study.

\section{Results}

There were 120 patients assessed for eligibility in the present study, out of which 38 were excluded due to nonadherence to inclusion and exclusion criteria. These also included fresh cases of Kala-azar without history of any anti-Kala-azar treatment. Thus only 82 patients were randomly assigned to the intervention.

A total of 82 SSG unresponsive and parasitologically confirmed VL cases were divided randomly into two groups before the initiation of the treatment. These were included in the study (41 receiving Amphotericin B and 41 receiving pentamidine). The clinical and laboratory characteristics of the patients of both the treatment groups were compared and there were no significant differences $(p>0.05)$ in the baseline characteristics of patients. The two groups were comparable (Table 1).
Table I Clinical and laboratory characteristics of two study groups before treatment

\begin{tabular}{|c|c|c|c|}
\hline & $\begin{array}{l}\text { Study group } \\
\text { Mean } \pm \text { SD }\end{array}$ & $\begin{array}{l}\text { Control group } \\
\text { Mean } \pm \text { SD }\end{array}$ & p-value \\
\hline No. of patients & $4 \mid(M=27, F=14)$ & $4 \mid(M=26, F=15)$ & \\
\hline Age & $18.3 \pm 11.3$ & $17.8 \pm 11.0$ & $>0.05$ \\
\hline \multicolumn{4}{|l|}{ Clinical } \\
\hline Weight (Kg) & $30.02 \pm 12.23$ & $28.55 \pm 12.28$ & $>0.05$ \\
\hline Fever $\max (\mathrm{F})$ & $101 \pm 1.64$ & $101.5 \pm 1.57$ & $>0.05$ \\
\hline Spleen size $(\mathrm{cm})$ & $7.79 \pm 3.7$ & $7.64 \pm 3.3$ & $>0.05$ \\
\hline Liver size $(\mathrm{cm})$ & $2.56 \pm 1.45$ & $2.8 \pm 1.75$ & $>0.05$ \\
\hline \multicolumn{4}{|l|}{ Laboratory } \\
\hline $\begin{array}{l}\text { WBC count } \\
(1000 / \mathrm{mm} 3)\end{array}$ & $4519 \pm 2230$ & $4004 \pm|74|$ & $>0.05$ \\
\hline $\begin{array}{l}\text { Platelet count } \\
\left(100,000 / \mathrm{mm}^{3}\right)\end{array}$ & $1.43 \pm 0.59$ & $1.29 \pm 0.44$ & $>0.05$ \\
\hline $\mathrm{Hb}(\mathrm{g} / \mathrm{dl})$ & $7.43 \pm 1.63$ & $6.94 \pm 1.37$ & $>0.05$ \\
\hline BSF (mg/dl) & $75 \pm 9.77$ & $73 \pm 8.26$ & $>0.05$ \\
\hline $\operatorname{BSPP}(\mathrm{mg} / \mathrm{dl})$ & $93 \pm 9.79$ & $90 \pm 9.45$ & $>0.05$ \\
\hline
\end{tabular}

Abbreviations: BSF, blood sugar (fasting); BSPP, blood sugar (post-parental); WBC, white blood cell.

All the clinical and laboratory characteristics of patients in each group were compared at the end of the treatment. There was significant changes in all clinical and laboratory characteristics in the Amphotericin B group except in blood sugar level, SGPT, creatinine, and Bilirubin, whereas in the Pentamidine group there was a significant increase in blood sugar level. The two groups were comparable (Table 2).

Apparent cure rate in the Amphotericin B group was found to be $95 \%$ (39/41) compared with $83 \%(34 / 41)$ in the Pentamidine group, which shows a significant statistical difference $(p=0.05)$. All the patients were followed up for six months after the end of treatment for recording relapse in two groups. In the Pentamidine group, four patients relapsed after 2-4 months and were treated with AMB, whereas only one case relapsed after two months in the Amphotericin B group. The ultimate cure rate was found $92 \%(38 / 41)$ in the Amphotericin B group as compared to $73 \%(30 / 41)$ in the Pentamidine group at the end of follow-up, (Table 3) which shows a significant statistical difference in the cure rate of AMB as compared to pentamidine $(p=0.04)$. Similarly, a statistically significant difference was observed in the relapse rate of the Amphotericin B group compared to the Pentamidine group $(p=0.03)$.

In the course of follow-up, eight patients did not turn up for follow-up on their scheduled time. Of these, four came after receiving the postcards sent by the Institute. 
Table 2 The clinical and laboratory features of the treatment group after the end of treatment are presented

\begin{tabular}{|c|c|c|c|c|c|c|}
\hline \multirow{3}{*}{ Characteristics } & \multicolumn{3}{|c|}{ Amphotericin group } & \multicolumn{3}{|c|}{ Pentamidine group } \\
\hline & Before $t / t$ & After $\mathbf{t} / \mathbf{t}$ & p-value & Before $t / t$ & After $\mathbf{t} / \mathbf{t}$ & p-value \\
\hline & Mean \pm SD & Mean \pm SD & & Mean \pm SD & Mean \pm SD & \\
\hline \multicolumn{7}{|l|}{ Clinical } \\
\hline Weight (Kg) & $30.02 \pm 12.23$ & $36.3 \pm 12.13$ & $<0.05$ & $25.55 \pm 12.28$ & $26.96 \pm 12.34$ & $<0.05$ \\
\hline Fever max $(F)$ & $10 \mathrm{I} \pm 1.64$ & $97.9 \pm 0.79$ & $<0.001$ & $101.5 \pm 1.57$ & $98.4 \pm 1.65$ & $<0.01$ \\
\hline Spleen size $(\mathrm{cm})$ & $7.79 \pm 3.7$ & $2.05 \pm 2.24$ & $<0.01$ & $7.05 \pm 2.9$ & $2.73 \pm 2.62$ & $<0.01$ \\
\hline Liver size $(\mathrm{cm})$ & $2.56 \pm 1.45$ & $0.58 \pm 0.91$ & $<0.01$ & $2.8 \pm 1.95$ & $0.85 \pm 1.15$ & $<0.01$ \\
\hline \multicolumn{7}{|l|}{ Laboratory } \\
\hline WBC count $\left(1000 / \mathrm{mm}^{3}\right)$ & $4519 \pm 2230$ & $6870 \pm 2141$ & $<0.01$ & $4004 \pm 174 \mid$ & $6163 \pm 2372$ & $<0.01$ \\
\hline Platelet count $\left(100,000 / \mathrm{mm}^{3}\right)$ & $1.43 \pm 0.59$ & $1.97 \pm 0.59$ & $<0.01$ & $1.29 \pm 0.44$ & $1.79 \pm 0.48$ & $<0.01$ \\
\hline $\mathrm{Hb}(g / d l)$ & $7.43 \pm 1.63$ & $8.96 \pm 1.4 \mid$ & $<0.01$ & $6.94 \pm 1.37$ & $8.34 \pm 1.31$ & $<0.01$ \\
\hline BSF (mg/dl) & $75 \pm 9.77$ & $76 \pm 8.79$ & $>0.05$ & $73 \pm 8.26$ & $77.45 \pm 9.97$ & $<0.05$ \\
\hline BSPP (mg/dl) & $93 \pm 9.79$ & $94 \pm 8.56$ & $>0.05$ & $90 \pm 9.45$ & $95 \pm 9.66$ & $<0.05$ \\
\hline SGPT (units/L) & $22.05 \pm 16.96$ & $18.96 \pm 12.14$ & $>0.05$ & $22.07 \pm 5.62$ & $19.38 \pm 6.08$ & $<0.05$ \\
\hline SGOT (units/L) & $24.59 \pm 5.61$ & $19.09 \pm 11.78$ & $<0.05$ & $22.23 \pm 6.88$ & $17.69 \pm 14.77$ & $>0.05$ \\
\hline Creatinine $(\mathrm{mg} / \mathrm{dl})$ & $0.67 \pm 0.31$ & $0.623 \pm 0.28$ & $>0.05$ & $0.65 \pm 0.15$ & $0.58 \pm 0.21$ & $>0.05$ \\
\hline Bilirubin (mg/dl) & $0.6 \pm 0.55$ & $0.545 \pm 0.3$ & $>0.05$ & $0.55 \pm 0.1$ & $0.51 \pm 0.12$ & $>0.05$ \\
\hline
\end{tabular}

Abbreviations: BSF, blood sugar (fasting); BSPP, blood sugar (post-parental); SGOT, serum glutamic oxaloacetic transaminase; SGPT, serum glutamic pyruvic transaminase; WBC, white blood cell.

The remaining four were attended by the hospital staff by making a home visit to their houses for follow-up. All the patients completed the follow-up for six months.

In the Amphotericin B group, two patients developed mild elevation of creatinine and blood urea nitrogen (BUN). This was ascertained on the basis of their blood urea and creatinine investigation, which was performed after infusion of seven injections. Here the mild elevation is of grade-II, therefore the treatment was discontinued for a period of seven days and as soon as these changes became normal, AMB was restarted. After a full course of AMB, the patient was found apparently cured. On the other hand, five patients developed hypoglycemia during the course of administering the drug in the Pentamidine group, which was treated with $25 \%$ dextrose infusion. It was ascertained on the basis of their blood sugar level, which was performed on 0, 7 days at the end of treatment, and during follow-up. The data is presented in Table 1. Blood sugar was done to rule out the diabetes mellitus for the pentamidine regimen. No significant abnormalities in liver function tests (serum glutamic pyruvic transaminase [SGPT], serum glutamic oxaloacetic transaminase [SGOT], serum Bilirubin) and renal function tests (blood urea and serum creatinine) were observed during the treatment (hence midterm evaluation of hematological and biochemical markers were not presented in Table 2). This was ascertained on the basis of their seventh day investigations after infusion during the treatment. Patients not apparently cured at the time of end of treatment were managed with a second course of AMB (rescue treatment) after an interval of 15 days. No deaths occurred in this trial.

\section{Discussion}

In the present study, the apparent cure rate of AMB was $95 \%$ as compared to $83 \%$ in the pentamidine group, which shows a statistically significant difference $(p=0.05)$. The apparent cure rate found under the present study was consistent with the finding of even later conducted studies. ${ }^{12,22}$ A study conducted in 2004 in Iran revealed the efficacy of AMB was up to $100 \% .^{25}$ In another study conducted in 2007 , the efficacy of AMB was $97 \%{ }^{26}$ The ultimate cure rate of AMB was $92 \%$ without any significant toxicity, which shows consistent efficacy over a period of time as was observed in

Table 3 Response of treatment with two regimens

\begin{tabular}{llll}
\hline Response & $\begin{array}{l}\text { Amphotericin-B } \\
(\mathbf{N}-4 \mathrm{I})\end{array}$ & $\begin{array}{l}\text { Pentamidine } \\
\mathbf{( N - 4 I )}\end{array}$ & $\begin{array}{l}\text { Chi-square } \\
\text { (p value) }\end{array}$ \\
\hline a. Apparent cure & $95 \%(39 / 4 \mathrm{I})$ & $83 \%(34 / 4 \mathrm{I})$ & $\mathrm{P}=0.05$ \\
b. Ultimate cure & $92 \%(38 / 4 \mathrm{I})$ & $73 \%(30 / 4 \mathrm{I})$ & $\mathrm{P}=0.04$ \\
c. Relapse rate & $2.5 \%(\mathrm{I} / 39)$ & $\mathrm{II} .7 \%(4 / 34)$ & $\mathrm{P}=0.03$ \\
\hline
\end{tabular}


earlier studies. ${ }^{17,18,27}$ The ultimate cure rate of pentamidine was $73 \%$, which may be the lowest ever reported in Bihar with significant toxicity and increased relapse rate $(12 \%)$, which is statistically significant $(\mathrm{p}=0.04)$. This finding was consistent with later conducted studies. ${ }^{10-12}$

The efficacy of AMB and its lipid formulation was found equally effective ${ }^{28}$ whereas the efficacy of AMB lipid formulation was found as high as $100 \%{ }^{20}$ which further declined to $92 \%$ in a study conducted in $2007 .{ }^{26}$

The average cost of a full course of AMB for adults comes to around US\$69 which is much less than the cost of the lipid formulation (US\$1120 for multiple infusions and US\$560 for single infusion). ${ }^{26}$ The cost of pentamidine is also much higher than a full course of AMB as it is imported. Initially in the $1950 \mathrm{~s}$, the cure rate of pentamidine was $99 \% 0^{29}$ but over a period of time in the year 1994, the reported cure rate of pentamidine was $82 \%$ in Bihar, ${ }^{30}$ which shows a gradual declining trend. The failure of pentamidine in the treatment of SSG-unresponsive Kala-azar cases posed a serious problem, because it was considered as a second-line drug, and was widely used in district hospitals in Kala-azar endemic areas of Bihar. Similarly, a statistically significant difference was observed in the relapse rate of the Amphotericin B group $(2.5 \%)$ compared to the Pentamidine group $(11.7 \%)(\mathrm{p}=0.03)$. The relapse rate of $\mathrm{AMB}$ found in this study was slightly higher compared to an earlier conducted study $(1.5 \%){ }^{31}$

Clinical and laboratory characteristics of the patients of both the treatment groups were compared and no statistically significant difference $(\mathrm{p}>0.05)$ in the base line characteristics were found. At the end of treatment significant changes were observed in all clinical and laboratory characteristics in the Amphotericin B group except in blood sugar level, SGPT, creatinine, and Bilirubin whereas in the Pentamidine group, there was significant increase in blood sugar level, which was remarkably consistent to several other studies conducted in Bihar. ${ }^{8,9}$ The relapse rate $(2.5 \%)$ of the Amphotericin B group showed a significant difference compared to (12\%) the Pentamidine group. It indicates that the relapse rate of pentamidine is increasing over a period of time, which was similar to other study findings. ${ }^{32}$ On the other hand, the relapse rate of AMB has remained consistent and within the acceptable range. In our study, we had found two patients (2/41) who developed mild elevation of creatinine and BUN and therefore treatment was discontinued for a period of seven days. As soon as these changes became normal, AMB was restarted. On the other hand, in the Pentamidine group, five patients (5/41) developed hypoglycemia while administering the drug and were treated with $25 \%$ dextrose infusion intravenously.
These finding were similar but not to the extent as reported earlier (7\%-9\% mortality and 60\% toxicity with irreversible damage such as diabetes and cardiac toxicity leading to fatal arrhythmias) in other studies. ${ }^{23,27}$ The study incorporates the valuable use of AMB in the treatment of antimony unresponsive Kala-azar cases. Several workers suggested using alternative drugs especially in cases of SSG-unresponsive and relapsing patients in its recommended dosage. ${ }^{17,32}$ Therefore treatment of Kala-azar with SSG and pentamidine has posed a major therapeutic challenge in combating Kala-azar in Bihar. Pentamidine acts against parasites by damaging kinetoplast DNA-mitochondrial complex. ${ }^{9}$ AMB on the other hand has the ability to inhibit biosynthesis of ergo sterol like steroid membrane sterols for both fungi and leishmania, thereby making it a potent antileishmanial drug. ${ }^{23}$

There is a need to discuss the therapeutic advances in VL to realize the difficulties occurring in the management of Kala-azar patients in Bihar. The newer drug, miltefosine, appears to be a step forward for Kala-azar treatment, but the major drawbacks are its effect on the fetus, which restricts its use in childbearing age, during the course of treatment, and after two months of completion of therapy. Hence $30 \%$ among the female population of the childbearing age (15-45 yrs) will be excluded from treatment on these grounds. The other drawback is its unaffordable price. ${ }^{33}$ Therefore it cannot be used as a mass outpatient treatment. On the other hand, an earlier study revealed that AMB could be effectively used during pregnancy with no harmful effects on the fetus. ${ }^{34}$ Another study on AIDS-associated VL reported a satisfactory response to AMB. ${ }^{21}$ Another study found that AMB efficiently responded in patients unresponsive to pentamidine. ${ }^{35}$ More recently, a clinical trial of miltefosine vs AMB reported a cure rate (after six months follow-up) of $94 \%$ and $97 \%$, respectively. ${ }^{36}$ Therefore, at present AMB has taken the lead in the treatment of Kala-azar.

From the days of Dr U.C. Brahamachari, various researchers hailing from Bihar have been continuously making efforts to conduct clinical trials of antileishmanials. Regular monitoring of efficacy of various antileishmanials in the form of clinical trials have proved to be very beneficial in addressing problems like unresponsiveness to SSG and pentamidine, which were considered the drug of choice 15 years ago. Introducing AMB as a first-line drug in the national Kalaazar elimination programme (2001) had significant impact on the management of Kala-azar treatment in India. Insufficient treatment from unqualified providers and widespread lack of compliance emphasizes the need for intensive community education and better access to health services. 
The results of this study suggest that AMB with an ultimate cure rate of $92 \%$ and with a relapse rate of $2.5 \%$ is still the only valuable drug of choice for the treatment of Kala-azar in Bihar. The issue of treatment of VL in developing countries is no longer limited to an individual patient. It is a public health problem considering that inadequate and ineffective therapy is fraught with emergence of secondary resistance. The issue of controlling VL also goes beyond development of new effective drug and therapies and implementation of vector control measures. The world has to face the problem of high costs of therapy squarely as control of VL cannot wait till the economic situation improves and social and cultural issues tackled.

The socioeconomic and cultural conditions of Bihar have a tremendous bearing on the prevalence of Kala-azar and its treatment. In practice most of the patients are expected to purchase the drug by themselves which leads to low compliance to the therapy. Hence the Indian Government is supposed to initiate steps to provide free/subsidized care and simultaneously ensure the regular availability of drug at peripheral level.

Considering the importance of AMB for Kala-azar patients, every effort should be taken to form a future strategy so that this drug and coming newer drugs do not meet a similar fate to SSG and pentamidine in a span of 10-15 years. The consistent efficacy of AMB over a period of time and relative cost effectiveness for treatment of SAG-unresponsive cases, even in pregnant women, in Bihar, show it to be a good choice. Generally, AMB is being used in public health system in Bihar even at periphery level (district hospital/primary health center) and the toxicity is being managed at the periphery level by the availability of pathological laboratory on public/private partnership in Bihar.

\section{Conclusion}

Amphotericin B appears to be a very efficient drug in the armamentarium of clinicians for the treatment of SSGunresponsive Kala-azar cases. Thus the findings of this study have important implication for health policy makers for the recent Kala-azar elimination programme (2001) in India.

\section{Disclosure}

The authors report no conflicts of interest in this work.

\section{References}

1. Murray HW. Clinical and experimental advances in treatment of visceral leishmaniasis. Antimicrob Agents Chemother. 2001;45:2185-2197.

2. Guerin P, Olliaro P, Sundar S, et al. Visceral leishmaniasis: current status of control, diagnosis, and treatment, and a proposed research and development agenda. Lancet Infect Dis. 2002;2:494-501.
3. Arias JR, Monteiro PS, Zicker F. The re-emergence of visceral leishmaniasis in Brazil. Emerg Infect Dis. 1996;2:145-146.

4. Ranjan A, Sur Dipika Singh VP, Siddiqui NA, et al. Risk factors for Indian Kala-azar. Am J Trop Med Hyg. 2005;73:74-78.

5. Sundar S, More DK, Singh MK, et al. Failure of Pentavalent antimony in visceral leishmaniasis in India: report from the center of Indian epidemic. Clin Infect Dis. 2000;31:1104-1106.

6. Thakur CP, Narayan S. A comparative evaluation of Amphotericin B and Sodium Antimony Gluconate, as first line drugs in the treatment of Indian visceral leishmaniasis. Ann Trop Med Parasitol. 2004;98:129-138.

7. Das VNR, Ranjan A, Bimal S, et al. Magnitude of unresponsiveness to Sodium-Stibo-Gluconate in the treatment of visceral leishmaniasis in Bihar. Natl Med J India. 2005;18:131-133.

8. Mishra M, Biswas UK, Jha AM, Khan AB. Amphoterocin B versus Pentamidine in antimony-unresponsive Kala-azar cases. Lancet. 1992;340:1256-1257.

9. Das VNR, Ranjan A, Sinha AN, et al. A randomised clinical trial of low dosage combination of pentamidine and allopurinol in the treatment of antimony unresponsive cases of visceral leishmaniasis. J Assoc Physicians India. 2001;49:609-612.

10. Olliaro PL, Guerin PJ, Gersti S, Haaskjold AA, Rottingen JA, Sundar S. Treatment options for visceral leishmaniasis: a systematic review of clinical studies done in India, 1980-2004. Lancet Infect Dis. 2005;5:763-774.

11. Croft SL, Sundar S, Fairlamb AH. Drug resistance in leishmaniasis. Clin Microbiol Rev. 2006;19:111-126.

12. Sundar S, Chatterjee M. Visceral Leishmaniasis- current therapeutic modalities. Indian J Med Res. 2006;123:345-352.

13. Berman JD. Human Leishmaniasis: clinical, diagnostic and chemotherapeutic development in the last 10 years. Clin Infect Dis. 1997;24:684-703.

14. Murray HW. Treatment of visceral leishmaniasis (Kala-azar): a decade of progress and future approaches. Int $J$ Infect Dis. $2000 ; 4: 158-177$.

15. Olliaro PL, Bryceson ADM. Practical progress and new drugs for changing patterns of leishmaniasis. Parasitol Today. 1993;9:323-328.

16. Bhattacharya SK, Sinha PK, Sundar S, et al. Phase 4 trial of miltefosine for the treatment of Indian visceral leishmaniasis. J Infect Dis. 2007;196:591-598.

17. Mishra M, Biswas UK, Jha AM, Khan AB. Amphotericin versus sodium stibo-gluconate in first line treatment of Indian Kala-azar. Lancet. 1994;344:1599-1600.

18. Thakur CP, Sinha SP, Sharma V, Pandey AK, Kumar M, Verma BB. Evaluation on Amphotericin B as a first line drug in comparison to Stibo-Gluconate in the treatment of fresh cases of Kala-azar. Indian $J$ Med Res. 1993;97:170-175.

19. Thakur CP, Kumar P, Kumar N, Singh GN, Kumar A, Narayan S. A randomised comparison of classical mode of Amphotericin B with its newer modes of administration in Kala-azar. $J$ Assoc Physicians India. 1998;46:762-765.

20. Thakur CP. A single high dose treatment of Kala-azar with Ambisome (Amphotericin B Lipid complex): a pilot study. Int J Antimicrob Agents. 2001;17:67-70.

21. Bernard E, Quaranta JF, Durant J, Le Fichous Y, Dellamonica P. Visceral Leishmaniasis resistant to conventional treatments: value of Amphotericine B. Pathol Biol (Paris). 1993;41(8 Pt. 2):817-819.

22. Sundar S, Chakravarty J, Rai VK, et al. Amphotericin B treatment for Indian visceral leishmaniasis: Response to 15 daily versus alternate day infusions. Clin Infect Dis. 2007;45:556-561.

23. Sundar S. Management of Kala-azar in India: Current Concepts. 2005. Cited Oct 10, 2008. Available from: http://medind.nic.in/imvw/ imvw6472.html.

24. Thakur CP, Singh RK, Hassan SM, Kumar R, Narain S, Kumar A. Amphotericin B deoxycholate treatment of visceral leishmaniasis with newer modes of administration and precautions: a study of 938 cases. Trans R Soc Trop Med Hyg. 1999;93:319-323. 
25. Tabatabaie P, Saidati A, Khotaii G, Manishi S. Successful treatment with amphotericin B of patients with visceral Leishmaniasis resistant to meglumine antimonite. Acta Medica Iranica. 2004;42:61-64.

26. Vanlerberghe V, Diap G, Guerin PJ, et al. Drug policy for visceral Leishmaniasis: a cost effectiveness analysis. Trop Med Int Health. 2007;12:274-283.

27. Thakur CP, Sinha GP, Pandey AK, et al. Do the diminishing efficacy and increasing toxicity of SSG, in the treatment of Kala-azar in Bihar, India, justify its continued use. Ann Trop Med Parasitol. 1998;92:561-569.

28. Sundar S, Mehta H, Suresh AB, Singh P, Murray HW. Amphotericin B treatment for Indian Visceral Leishmaniasis: conventional verses lipid formulation. Clin Infect Dis. 2004;38:377-383.

29. Jha TK. Evaluation of Pentamidine isethionate in the treatment of Kala-azar occurring in North-Bihar, India. Trans R Soc Trop Med Hyg. 1983;77:167-170.

30. Sinha AN, Sinha SP, Sen AB, Saran R. Unresponsiveness in Indian Kala-azar. Indian J Parasitol. 1994;18:7-12.
31. Sundar S, Agrawal G, Rai M, Makharia MK, Murray HW. Treatment of Indian visceral leishmaniasis with single or daily infusions of low dose liposomal Amphotericin B: randomized trial. $\mathrm{Br}$ Med J. 2001;323:419-422.

32. Thakur CP, Kumar MM, Pandey AK. Comparisons of regimens of treatment of resistant Kala-azar patients: A randomised study. Am J Trop Med Hyg. 1991;45:435-440.

33. Rosenthal E, Marty P. Recent understanding in the treatment of visceral leishmaniasis. J Postgrad Med. 2003;49:61-68.

34. Thakur CP, Sinha GP, Sharma V, Barat D. Treatment of Kala-azar during pregnancy. Natl Med J India. 1993;6:263-265.

35. Giri OP. Treatment of Visceral Leishmaniasis unresponsive to Pentamidine with Amphotericin B. J Assoc Physicians India. 1994;42:688-689.

36. Sundar S, Jha TK, Thakur CP, et al. Oral miltefosine for Indian leishmaniasis. N Engl J Med. 2002;347:1739-1746. 